DOI: 10.12731/2227-930X-2019-1-42-50

УДК 004.94

\title{
ЭКСПЕРТНО-ОПТИМИЗАЦИОННОЕ МОДЕЛИРОВАНИЕ В ПРОЦЕССАХ ПЕРЕВОЗОК
}

\section{Львович Я.Е., Преображенский А.П., Чопоров О.Н.}

В данной статье рассматриваются вопросы, связанные с ранжированием объектов, связанных с перевозками, по областям. Дано описание оптимизационной модели и экспертного подхода. Приведена структурная схема, иллюстрирующая процедуру экспертно-оптимизачионного моделирования.

Ключевые слова: система перевозок; экспертное оченивание; оптимизачия; моделирование; организация.

\section{EXPERT-OPTIMIZATION MODELING IN TRANSPORT PROCESSES}

\section{Lvovich Ya. E., Preobrazhenskiy A.P., Choporov O.N.}

This paper discusses issues related to the ranking of objects related to transportation, by region. The description of the optimization model and expert approach is given. A block diagram illustrating the procedure of expert optimization modeling is presented.

Ключевые слова: transportation system; expert evaluation; optimization; modeling; organization.

\section{Введение}

Когда рассматривается управление процессами перевозок в организациях в ряде случаев ориентируются на показатели рейтингово-мониторингового оценивания $[1,2]$. Объекты в системе перевозок могут быть разделены различным образом по областям, в зависимости от их характеристик [3]. Для того, чтобы выбрать 
оптимальный вариант, необходимо использовать комбинацию оптимизационных методов и экспертных оценок.

В работе авторами предлагается комбинированный подход позволяющий осуществить по областям объекты в системе перевозок.

\section{Описание задачи перевозок}

Предположим, что на базе мониторинго-рейтингового оценивания осуществлено ранжирование объектов $O_{i}\left(i=1^{\prime}, I\right)$, которые, могут быть, например, организациями в системе перевозок относительно величины $R_{i}\left(i=1^{\prime}, l\right)$. Внутри числового интервала [A, O] осуществляется ее нормировка. В управляющем центре сетевых перевозок эксперты определяют число областей (М). По ним распределяются объекты системы перевозок [4]. Экспертным путем задаются границы соседних областей. Максимальная величина интегральной оценки А будет им пропорциональна:

$$
R_{m-1, m}=v_{m-1, m} A \text {. }
$$

Коэффициент пропорциональности в (1) - это $v_{m-1, m}$. Роль его заключается в разделении между ( $m-1)$-й и $m$-й областью, $0<v_{m-1, m}<1$.

Это будет нормативным разделением. С тем, чтобы оно было верифицировано по множеству объектов $O_{i}\left(i=1^{\prime}, I\right)$ при $\left.m=1^{\prime}, M\right)$ областях, нами предложено формирование оптимизационной модели.

Предположим, что когда есть разделение при условии (1) в области $(m-1)$-ю и $m$-ю будут входить объекты $O_{\mathfrak{\Im}-1, m}\left(i_{m-1, m}=1, I_{m-1, m}^{\prime}\right)$. Проведение оптимизационного моделирования мы осуществляем за счет того, что вводятся альтернативные переменные:

$$
x_{i}=\left\{\begin{array}{c}
1, \text { еслиі-й объект будет в }(m-1)-\text { й области }, \\
0, \text { в противном случае } \\
i=1, I_{m-1, m}^{\prime} .
\end{array}\right.
$$

Следует проводить так выбор по значениям переменных (2), чтобы соблюдалось следующее условие. Внутри области, которая находится на более высоком уровне, располагают объекты, у которых большие значения интегральных оценок. 
Идет определение по множеству показателей со стороны управляющего центра $y_{i}=1^{\prime}, J$. Среди них некоторого количество $s=1^{\prime}, S$ базовых. На основе них можно установить пороговые значения $y_{s}^{n}\left(s=1^{\prime}, S\right)$. Происходит формирование набора двоичных оценок по каждому из $i-i \mathrm{x}$ объектов

$$
c_{s i}=\left\{\begin{array}{c}
1, \text { если } y_{i s} \geq y_{s}^{n}, \\
0, \text { в противном случае }, s=1, s .
\end{array}\right.
$$

Если рассматривать правило большинства [5] работа объекта может быть охарактеризована некоторыми требованиями эффективности, когда

$$
\sum_{s=1}^{S} c_{s i}>\frac{1}{2} S .
$$

Существуют такие объекты в $(m-1)$-й и $m$-й областях, по которым нет выполнение условия (3). В этой связи, когда такие области разделяются, мы можем опираться на условие, являющимся более слабым

$$
\sum_{s=1}^{S} c_{s i}>C_{m-1, m}<\frac{1}{2} S
$$

Проведение выбора объектов происходит внутри оптимизационной модели. Происходит в виде ограничений запись условия (4):

$$
\begin{gathered}
\sum_{i=1}^{I_{m-1, m}} Y_{i} x_{i} \rightarrow \max ; \\
\sum^{S} c_{s i} x_{i} \geq C_{m-1, m}, i=1, I_{m-1, m}^{\prime}, \\
x_{i}=\left\{\begin{array}{c}
1,, i=1, I_{m-1, m} . \\
0
\end{array}\right.
\end{gathered}
$$

В [6] авторы продемонстрировали, что задачу (5) мы можем решить на базе того, что применяется направленный рандомизированный поиск. При этом вводятся вероятностные оценки $\tilde{x}_{i}$ переменных (2):

$$
P\left(\tilde{x}_{i}=1\right)=p_{x_{i}}, P\left(\tilde{x}_{i}=0\right)=q_{x_{i}}, p_{x_{i}}+q_{x_{i}}=1 .
$$

В качестве вероятности события обозначена $\mathrm{P}(\cdot)$. Также значениями вероятности события $\tilde{x}_{i}=1$ являются $p_{x_{i}}$. Значение вероят- 
ности события $\tilde{x}_{i}$ определяется на базе $q_{x_{i}}$ Требуется, чтобы выполнялись условия, когда осуществляется поиск, которые связанные с локальным улучшением каждых из $k-x$ итераций. В значениях вероятностей $p_{x_{i}}$ осуществляется изменение некоторых переменных. От значения 0,5 подобное изменение будет осуществляться к значениям, внутри окрестности $(1-\varepsilon, 1)$. При этом $0,5>\varepsilon>0$. Есть значения, которые будут в окрестности $(0, \varepsilon)$. В первом случае переменные рассматриваются как равные 1 :

$$
x_{i}=1, i=1, I_{1}
$$

а во втором - 0 :

$$
x_{i}=0, i=I_{1}+1, I_{m-1, m} .
$$

Тогда объекты $O_{i}, i=1^{\prime}, I_{1}$ могут быть отнесены к $(m-1)$-й области.

\section{Описание экспертного подхода}

Когда объекты распределяются среди ( $m-1)$-й и $m$-й областями, то это может не совпадать с тем, как это разделили эксперты в управляющем центре, может потребоваться и поиск компромиссного решения.

Эксперты опираются на способ, связанный с наглядно-образное описание [7]. Множества объектов $O_{i}, i=1, I^{\prime}{ }_{m-1, m}$ рассматриваются в виде изображений. На них видны многомерные мониторинговые оценки $y_{i j}, i=1, I_{m-1, m}^{\prime}, j=1^{\prime}, J$ в виде отдельных точек на плоскости [5]. Тогда эксперт по таким точкам может сделать заключение о границе между областями. Проводится изображение границы, которая получается на базе условия (1), по решению (6), а также с привлечением методик статистического (нейросетевого) моделирования того, как разделяются объекты по областям. Экспертом идет выделение точек, которые, будут в местах, где не совпадает эвристическое и оптимизационное решение. Они соответствуют объектам $O_{i}, i^{\prime}=1, I^{i} \in 1, I_{m-1, m}^{\prime}$. У экспертов кроме того, что есть мониторинго-рейтинговые оценки по объектам $O_{i}$, существуют возможности получения по ним дополнительной информации $[8$, 9]. Тогда они при учете (1) и применения статистических моделях могут проводить использование субъективных оценок 
$Z_{i}=\left\{\begin{array}{c}1, \text { когда объект } O_{i}^{\prime} \text { требуется отнести к }(m-1)-\text { й области }, \\ 0, \text { в противном случае }, i^{\prime}=1, I^{\prime} .\end{array}\right.$

Эксперты степень согласованности между решением (6) и оценкой

(7) по объектам $O_{i}$ характеризуют на основе значений вероятностей

$$
p_{z_{i}^{\prime}}=P\left(z_{i}^{\prime} / x_{i}^{\prime}=1\right), q_{i}^{\prime}=P\left(z_{i}^{\prime} / x_{i}^{\prime}=0\right) \text {. }
$$

Формула Байеса дает возможности для того, чтобы была коррекция по значениям вероятностей $p_{x_{i}^{\prime}}$

$$
P^{c k} x_{i}^{\prime}=\frac{p_{z_{i}} p_{x_{i}^{\prime}}}{p_{z_{i}} \cdot p_{x_{i}}+q_{z_{i}} q_{x_{i}^{\prime}}} ; i^{\prime}=1, I^{\prime} .
$$

Используется процедура рандомизированного поиска. При этом проходит процесс вычислений, использующий начальные значения вероятностей (9) по переменным $i^{\prime}=1, I^{\prime}$ и значениям $p_{x_{i}}^{k}$ по другим переменным. Продолжение процедуры происходит до того, как найден компромиссный вариант, который будет устраивать экспертов в управляющем центре. На рис. 1 приведена структурная схема, иллюстрирующая процедуру экспертно-оптимизационного моделирования того, как разделяются по областям объекты в системе перевозок.

\section{Выводы}

Чтобы проводить структуризацию объектов в системе перевозок по областям следует применять нейронные сети. Они позволяют учитывать изолированные области, которые, когда выполняется алгоритмическая процедура, не будут исчезать и не модифицируются. Необходимо проводить согласованное группирование объектов в системе перевозок относительно многомерных данных и интегральных оценок их эффективности и проведения упорядочения по областям.

При этом перспективным представляется использование определения альтернативных вариантов разделяющих границ среди областей за счет того, что применяется рандомизированный поиск компромиссного варианта.

Результаты экспертного оценивания формализуются на базе субъективных вероятностей, а также используются результаты нейросетевого моделирования. 


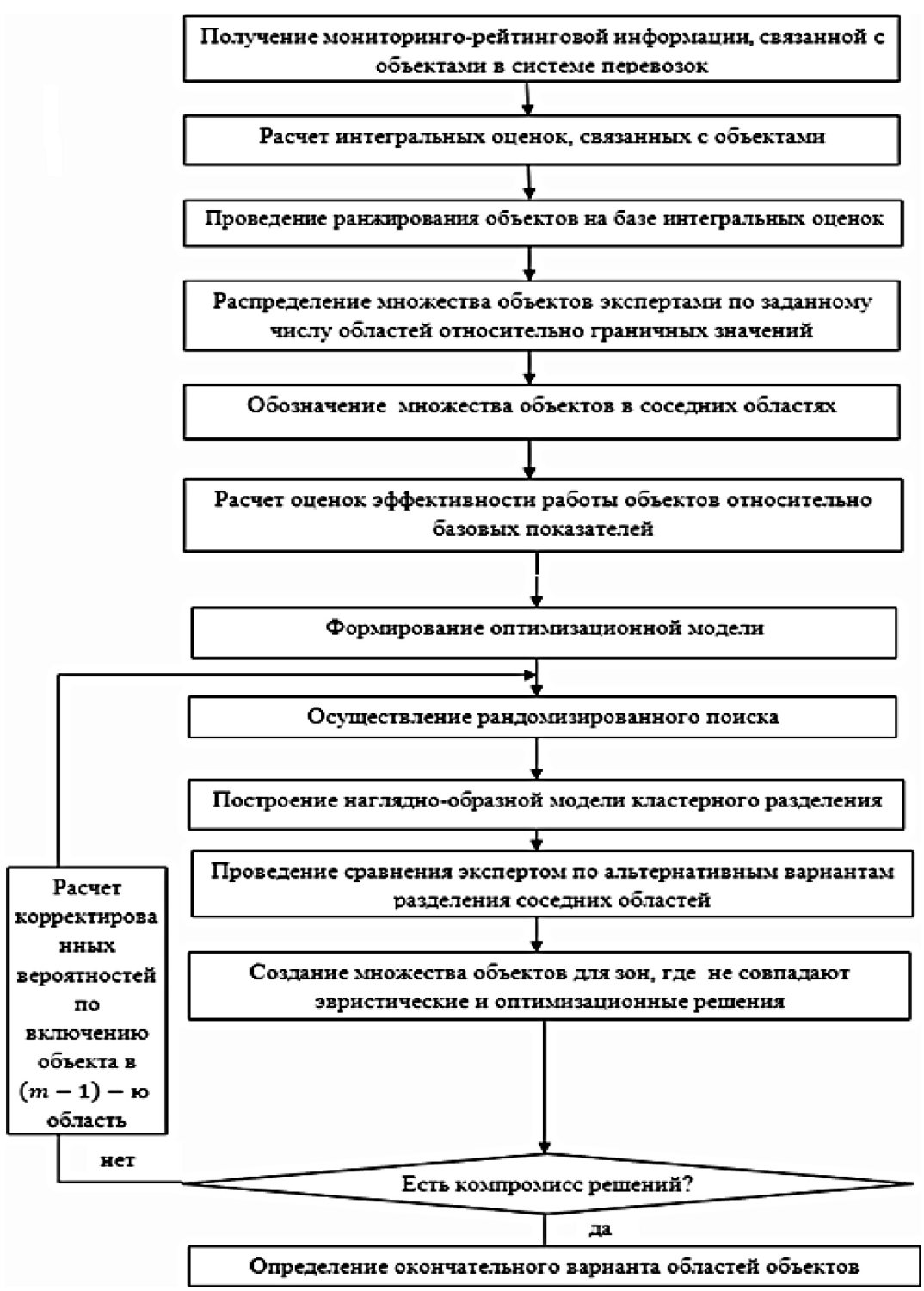

Рис. 1. Структурная схема, иллюстрирующая процедуру экспертно-оптимизационного моделирования разделения по областям объектов в системе перевозок 


\section{Список литературы}

1. Калиберда Е.А., Христосова Н.Г. Автоматизация деятельности отдела доставки заказов интернет-магазина // International Journal of Advanced Studies. 2018. T. 8. № 1-2. C. 65-72.

2. Тюльпинова Н.В. Программный модуль для имитационного моделирования предприятий розничной торговли // International Journal of Advanced Studies. 2018. T. 8. № 1-2. C. 187-192.

3. Юрочкин А.Г., Коростелева Н.А. Анализ особенностей управления и менеджмента в современных компаниях // Вестник Воронежского института высоких технологий. 2018. № 3(26). С. 78-80.

4. Гоян В.И., Никонова Е.3. Реинжиниринг и его место в жизненном цикле информационной системы // International Journal of Advanced Studies. 2019. T. 9. № 1-2. C. 45-51.

5. Львович Я.Е., Львович И.Я. Принятие решений в экспертно-виртуальной среде. Воронеж: Научная книга, 2010. 39 с.

6. Лотов А.В., Поспелова И.И. Многокритериальные задачи принятия решений. М.: МАКС Пресс, 2008. 197 с.

7. Литвак Б.Г. Экспертная информация. Методы получения и анализа. М. Радио и связь, 1982.184 с.

8. Зазулин А.В., Преображенский Ю.П. Особенности построения семантических моделей предметной области // Вестник Воронежского института высоких технологий. 2008. № 3. С. 26-28.

9. Зяблов Е.Л., Преображенский Ю.П. Построение объектно-семантической модели системы управления // Вестник Воронежского института высоких технологий. 2008. № 3. С. 29-30.

\section{References}

1. Kaliberda E.A., Hristosova N.G. International Journal of Advanced Studies. 2018. V. 8. № 1-2, pp. 65-72.

2. Tyul'pinova N.V. International Journal of Advanced Studies. 2018. V. 8. № 1-2, pp. 187-192.

3. Yurochkin A.G., Korosteleva N.A. Vestnik Voronezhskogo instituta vysokih tekhnologij. 2018. № 3(26), pp. 78-80.

4. Goyan V.I., Nikonova E.Z. International Journal of Advanced Studies. 2019. V. 9. № 1-2, pp. 45-51. 
5. L'vovich Ya.E., L'vovich I.Ya. Prinyatie reshenij v ekspertno-virtual'noj srede [Decision making in an expert-virtual environmen]. Voronezh: Nauchnaya kniga, 2010. 139 p.

6. Lotov A.V., Pospelova I.I. Mnogokriterial'nye zadachi prinyatiya reshenij [Multi-criteria decision making tasks]. M.: MAKS Press, 2008. 197 p.

7. Litvak B.G. Ekspertnaya informaciya. Metody polucheniya $i$ analiza [Expert information. Methods of obtaining and analysis]. M.: Radio i svyaz', 1982. 184 p.

8. Zazulin A.V., Preobrazhenskij Yu.P. Vestnik Voronezhskogo instituta vysokih tekhnologij. 2008. № 3, pp. 26-28.

9. Zyablov E.L., Preobrazhenskij Yu.P. Vestnik Voronezhskogo instituta vysokih tekhnologij. 2008. № 3, pp. 29-30.

\section{ДАННЫЕ ОБ АВТОРАХ}

Львович Яков Евсеевич, профессор, доктор технических наук, профессор

Федеральное государственное образовательное учреждение высшего образования «Воронежский государственный технический университет»

ул. 20 лет Октября, 84, г. Воронеж, 394006, Российская Федеращия

Komkovvivt@yandex.ru

Преображенский Андрей Петрович, профессор, доктор технических наук, доцент

Автономная некоммерческая образовательная организация высшего образования «Воронежский институт высоких технологий»

ул. Ленина, 73а, Воронеж, 394043, Российская Федерация Komkovvivt@yandex.ru

Чопоров Олег Николаевич, профессор, доктор технических наук Федеральное государственное образовательное учреждение выстего образования «Воронежский государственный технический университет» 
ул. 20 лет Октября, 84, г. Воронеж, 394006, Российская Федерация

Komkovvivt@yandex.ru

\section{DATA ABOUT THE AUTHORS}

Lvovich Yakov Yevseevich, Doctor of Technical Sciences, Professor Voronezh State Technical University

84, 20 let Oktyabrya Str., Voronezh, 394006, Russian Federation Komkovvivt@yandex.ru

ORCID: 0000-0002-7051-3763

Preobrazhenskiy Andrey Petrovich, Doctor of Technical Sciences, Professor, Associate Professor Voronezh Institute of High Technologies

73a, Lenin Str., Voronezh, 394043, Russian Federation

Komkovvivt@yandex.ru

ORCID: 0000-0002-6911-8053

Choporov Oleg Nikolaevich, Doctor of Technical Sciences, Professor Voronezh State Technical University 84, 20 let Oktyabrya Str., Voronezh, 394006, Russian Federation Komkovvivt@yandex.ru

ORCID: 0000-0002-3176-499X 